\title{
Analysis of COVID-19 Outbreak in Ecuador Using the Logistic Model
}

\author{
Talia Tene ${ }^{1}$, Marco Guevara ${ }^{2}$, Jiř́i Svozilík ${ }^{2,3}$, Richard Tene-Fernandez ${ }^{4}$, \\ Cristian Vacacela Gomez ${ }^{2 *}$ \\ ${ }^{1}$ Grupo de Investigación Ciencia y Tecnología de Materiales, Universidad Técnica Particular de Loja, EC-110160 Loja, Ecuador \\ ${ }^{2}$ School of Physical Sciences and Nanotechnology, Yachay Tech University, Urcuquí, EC-100119, Ecuador \\ ${ }^{3}$ Joint Laboratory of Optics of Palacký University and Institute of Physics of CAS, Faculty of Science, Palacký University, 17. \\ listopadu12, 77146 Olomouc, Czech Republic \\ ${ }^{4}$ Facultad de Ciencias Médicas, Universidad de Cuenca, EC-0101168 Cuenca, Ecuador
}

\begin{abstract}
At the end of 2019, the COVID-19 disease emerged in the city of Wuhan, China, and caused an outbreak of unusual viral pneumonia. Being highly transmissible, this novel coronavirus disease has spread fast all over the world. COVID-19 continues to challenge most developed countries in the search for an effective strategy to either prevent infection or to avoid the spreading of the disease. While several developed countries have managed to contain COVID-19, several countries in Latin America continue to report an increase in the daily number of infected people. Ecuador, particularly, became the epicenter of the COVID-19 outbreak in the region during March and April 2020. In this context, the present study shows a simple mathematical approach to understand the effect of the COVID-19 outbreak in Ecuador (and some Latin American countries such as Brazil, Peru, and Colombia). The proposed method is based on the exponential model, discrete logistic equation, and differential logistic model using one-year data from March 1, 2020, to February 28 , 2021. This study presents the estimated growth rate coefficient $(\lambda)$, the total number of cases $(\mathrm{N})$, and the midpoint of maximum infection $\left(\mathrm{t} \_0\right)$ as well as the variability of the $\lambda$ coefficient as a function of total cases and time. The exponential model shows a high value of $\lambda=0.185$ which decreases to $\lambda=0.014$ and $\lambda=0.056$ according to the discrete and differential logistic models, respectively. An accurate value of the total number of cases of infected people was found by analyzing the number of daily cases as a function of the total of cases whose value $(\mathrm{N} \sim 409 \mathrm{~K})$ agrees with the data reported at the end of May 2021, validating the proposed approach. How to use the current mathematical approach for long-term prediction is also discussed here. Most importantly, the proposed method has two important characteristics: (i) the mathematical model is as simple as possible compared to other time-consuming approaches, and (ii) it can be used to study the effect of COVID-19 and predicts its consequences in other countries, allowing revenue new decisions against the COVID-19 disease.
\end{abstract}

\section{Keywords:}

COVID-19;

SARS-CoV-2;

Logistic Model;

Mathematical Approach;

Ecuador.

Article History:

$\begin{array}{llll}\text { Received: } & 28 & \text { May } & 2021 \\ \text { Revised: } & 09 & \text { August } & 2021 \\ \text { Accepted: } & 17 & \text { August } & 2021 \\ \text { Published: } & 25 & \text { August } & 2021\end{array}$

\section{1- Introduction}

In December 2019 [1], a cluster of cases associated with a new respiratory syndrome was reported in China and spread rapidly around the world [2]. This syndrome is called coronavirus disease 2019 (COVID-19) [3] caused by a novel coronavirus SARS-COV-2, according to the International Committee on Taxonomy of Viruses [4]. The COVID-

*CONTACT: cvacacela@yachaytech.edu.ec

DOI: http://dx.doi.org/10.28991/esj-2021-SPER-09

(C) 2021 by the authors. Licensee ESJ, Italy. This is an open access article under the terms and conditions of the Creative Commons Attribution (CC-BY) license (https://creativecommons.org/licenses/by/4.0/). 
19 disease was declared a global pandemic on March 11, 2020, by the World Health Organization (WHO) [5] with more than $170 \mathrm{M}$ cases by the end of May 2021. Nowadays, the most affected five countries by the spread of COVID-19 are the US, India, Brazil, Russia, and France (see:https://www.worldometers.info/coronavirus/countries-where-coronavirushas-spread/). At this time, several countries of Latin America (e.g., Ecuador, Perú, Colombia) are also being harshly impacted by the current pandemic [6]. The unprecedented public health crisis created by the global spread of COVID19 presents an incomparable challenge all over Latin America [7].

Particularly, in Ecuador, COVID-19 has affected not only the public health system and emergency response but has also had profound socio-economic, equity, and ethical consequences. During this time, inexperience in the process of collecting, reporting, and analyzing data from public institutions became more evident, such complicating the appropriate decision-making of Ecuador's public health system, which resulted in a slow operational capacity and a lack of a strategic preparedness plan to mitigate the spread of the infection and cope with the increasing number of cases and deaths. With an overwhelmed health system, due to the high number of infected patients and delayed proactive response, Ecuador became the epicenter of the COVID-19 pandemic in Latin America during March and April 2020.

In this context, Alava et al. [8] indicated Ecuador's response capacity plan to counteract and mitigate the pandemic effect was not adequate to contain the transmission of the virus, especially in Guayas, Pichincha, Manabí, Azuay, and Tungurahua provinces. Moreover, the insufficiency of biosafety equipment for the frontline health personnel, the deficient number of trained staff, the critical care beds scarcity, and the unavailability of a coordinated plan for early detection conducted to the collapse of the Ecuadorian public health system. Torres et al. [9] examined the number of confirmed COVID-19 cases in Ecuador, which may have been influenced by the low capacity of laboratories to perform tests, underestimating or overestimating the real Ecuadorian situation. The latter suggests that it is necessary to augment laboratory capabilities for improved decision-making and related health policies. The impact of the COVID-19 outbreak in Ecuador was measured using the estimates of excess mortality [9]. Although, the study was limited to March 17October 22, 2020; the results evidenced excess mortality in Ecuador during April 4, 2020. In general, Ecuador had a high mortality rate $(882 \mathrm{pmp})$ by February 26,2021 . The country's positivity rate $(28 \%$ in 56,000 tests pmp) is higher than in other South American countries such as Uruguay (5.7\% in 290,000 tests pmp), Chile (8.8\% in 490,000 tests pmp), Colombia (19.6\% in 223,000 tests pmp), and even more than the US ( $8 \%$ in 1 million tests pmp) [10,11]. The Ecuadorian government has implemented social distancing, case testing, and random quarantine to protect the population against COVID-19 [12]. Nevertheless, these directives appear to be unsuccessful ineffective in controlling the disease to date. Taking all this into account, and also the novel virus mutation [13], the case of Ecuador represents an important challenge to understand the effect of COVID-19.

While the global urgency is to find a vaccine [14], the scientific community is using this crisis to identify the vulnerabilities by modeling reported data from the COVID-19 outbreak around the world to predict and prevent similar events. Then, precise estimations of the infection growth rate, turning point, duration of outbreaks, and total amount of COVID-19 cases, are common questions [15]. To address those questions, different mathematical approaches have been proposed, including models based on the simple logistic function [16], improved SIR and SEIR models [17], hierarchical polynomial regression models [18], statistical models including machine learning techniques [19], time-varying Markov process [20], Gaussian mixture model [21], among others. However, mentioned models should be used with caution, especially, if these models are used for long-term forecasting [22]. Recently, Pelinovsky et al. [23] reported a simple mathematical approach based on the logistic equation to study the COVID-19 outbreak in different countries (Austria, Switzerland, the Netherlands, Italy, Turkey and South Korea), considering the first months of the pandemic. In particular, the logistic model was considered appropriate for most countries, because it allowed to estimate the growth rate and the expected number of infected people. As well, Wang et al. [24] predicted the epidemic trends in COVID-19 using the epidemiological data before June 16, 2020 (for Brazil, Russia, India, Peru and Indonesia) employing the logistic model and machine learning time series prediction model. Abusam et al. [25] used the logistic model to describe the dynamics of COVID-19 pandemic in Kuwait based on the Verhulst and Richards approaches. Gao et al. [26] developed a mathematical model based on the logistic equation to provide a quantitative insight on the dynamics of age-specific casefatality rates. Aviv-Sharon et al. [27] provided a generalized logistic modeling to study the COVID-19 outbreak in Asia, demonstrating that the estimated parameters match the confirmed reports. From all these works it can be seen that the logistic model is an excellent option to explore the COVID-19 outbreak.

To the best of our knowledge, a one-year data analysis of the COVID-19 outbreak in Ecuador using the logistic model has not been reported yet. In this work, such a missing study is presented. Being more specific, the discrete logistic equation and ordinary differential equation (ODE) logistic model are used to study the Ecuadorian case as well as some Latin American countries, such as Brazil, Peru, Colombia. The proposed method allows estimating the infection growth rate, turning point, and the maximum number of cases. As infections and deaths continue throughout the world, this model can be extended to other countries or locations to take immediate corrective strategies due to it can be calibrated by adding the daily data of infected people and total cases as well. 


\section{2- Research Methodology}

\section{2-1- Data Collection and Analysis}

One-year data of COVID-19 outbreak in Ecuador, from March 1, 2020, to February 28, 2021, was taken (and compared) from:

- Worldometer (https://www.worldometers.info/coronavirus/country/ecuador/);

- Johns Hopkins Coronavirus Resource Center (https://coronavirus.jhu.edu/region/ecuador);

- Ministry of Public Health of Ecuador (https://www.salud.gob.ec/coronavirus-covid-19/).

From these sources, we have extracted the following values:

- The total number of coronavirus cases for given data;

- Daily news reported cases.

Some discrepancies were found between the given sources given, particularly, the first month of the pandemic. As mentioned, this can be attributed to the lack of qualified laboratories to carry out tests and large inexperience in collecting and reporting daily data. However, similar problems were also detected in the other Latin American countries studied here (Brazil, Peru, Colombia). For this reason, the present study uses a seven-point (7p) moving average (shown below), which means that it takes the last 7 days, adds them up, and divides them by 7 . This smooth-out resulting curves by canceling out peaks and valleys in data collection. For instance, if certain days, the laboratories might not be able to report their cases, but the following day yes, we will notice a combination of cases from the previous days and the current day in a report and such creating a large peak in data. However, using the 14-point and 21-point moving averages, it was observed that the estimated values could be underestimated. The fitting and needed computations were carried out on Mathematica 12 (license provided by CEDIA: https://www.cedia.edu.ec/es/licencias-wolfram).

All parameters considered in our models are:

- The total number of infected people as a function of time;

- The initial number of the infected people $t=0$ days;

- The maximum number of infected people;

- The point of maximum growth.

\section{2-2- Theoretical Approach}

We shall start with the following simple model. Exponential functions can be used to model population growth, interest rates, radioactive decay, and the amount of medicine in the bloodstream. Since our interest is to model the increasing number of infected people during the pandemic, the following equation is proposed:

$$
\frac{d N(t)}{d t}=\lambda_{\text {exp }} N(t)
$$

$\lambda_{\text {exp }}$ is taken as the (positive) exponential growth rate of the epidemic $\left(\right.$ day $\left.^{-1}\right)$ and $N(t)$ represents the total number of infected people which depends on time. Solving step-by-step Equation 1:

$$
\begin{aligned}
& \int \frac{d N(t)}{N(t)}=\int \lambda_{\text {exp }} d t \\
& \ln N(t)=\lambda_{\text {exp }} t+c \\
& N(t)=e^{\lambda_{\text {exp }} t} e^{c}
\end{aligned}
$$

One can finally get:

$$
N(t)=N_{0} e^{\lambda_{\exp } t}
$$

where $N_{0}$ is the initial number of the infected people and $t$ is the time since the first reported cases (day). Equation 5 can only be used at the early stage of the pandemic if the number of confirmed COVID-19 cases as a function of time is described by a clear exponential equation [28], which is illustrated in Figure 1a. In addition, during this initial period, we don't observe the depletion of possible infection candidates. 

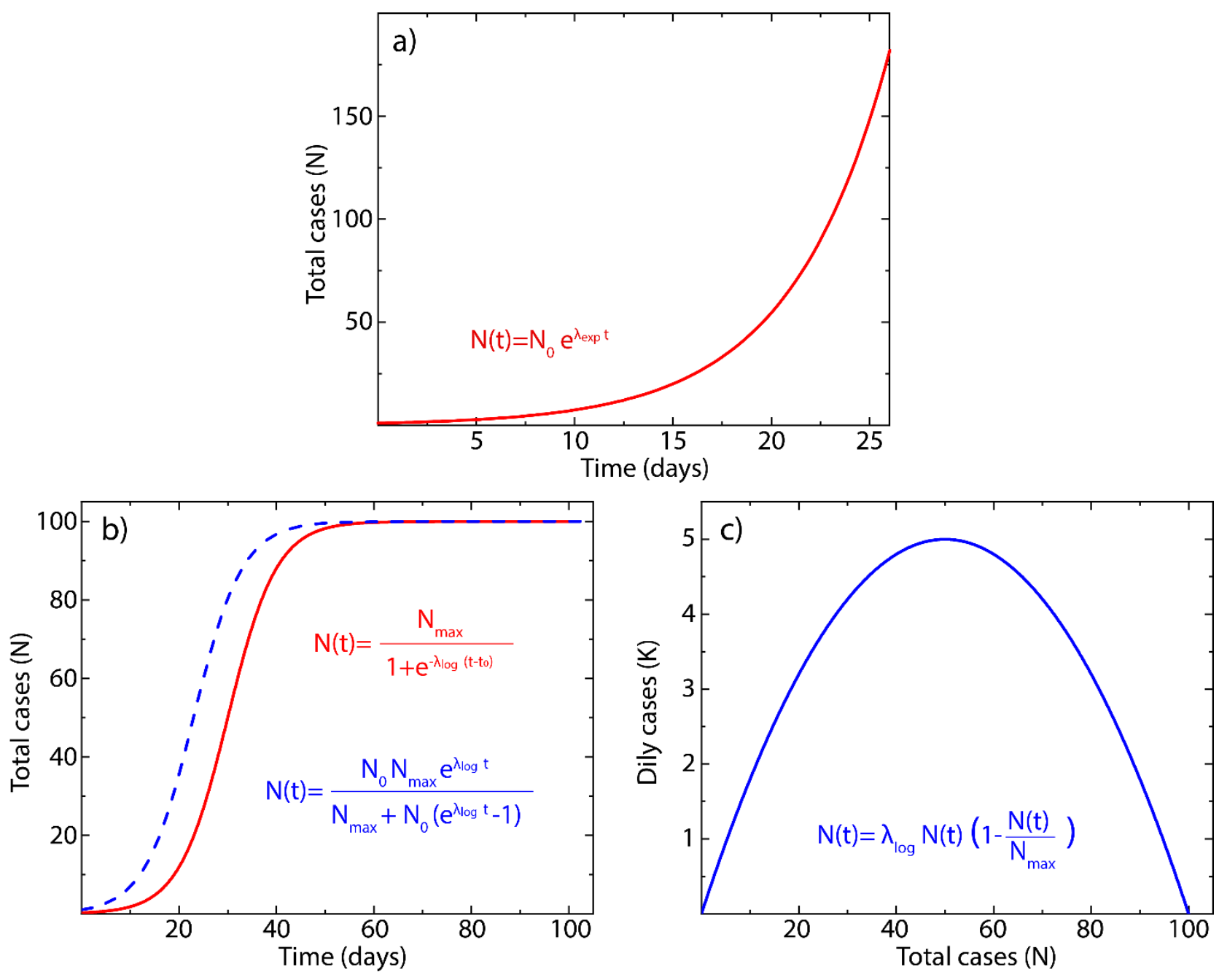

Figure 1. Illustration cases: a) the total number of cases as a function of time using the exponential model, b) the total number of cases as a function of time. The discrete logistic equations (red line) are compared to the solution of the ODE logistic model (dashed blue line), and c) the daily cases as a function of total cases. Parameters used: $N_{\max }=100, N_{0}=1, \lambda_{\log }=0.2\left(\right.$ day $\left.^{-1}\right)$, $\lambda_{\text {exp }}=0.2\left(\right.$ day $\left.^{-1}\right), t_{0}=30($ day $)$.

In fact, this model can be applied as long as there are no changes in the curve. On the other hand, if restrictions are implemented to flatten the curve (such as social distancing, case testing, hand washing, and quarantine), the dataset must be fitted using the discrete logistic equation [29]:

$N(t)=\frac{N_{\max }}{1+e^{-\lambda_{\log }\left(t-t_{0}\right)}}$

Which also allows estimating the maximum number of infected people $\left(N_{\text {max }}\right)$, the logistic growth rate $\left(\lambda_{\text {log }}\right.$, day $\left.{ }^{-1}\right)$, and the point of maximum growth $\left(t_{0}\right)$. Logistic functions were first studied in the context of population growth, as early exponential models failed after a significant amount of time had passed. By adding the correcting factor $-\frac{\lambda_{\log N(t)^{2}}}{N_{\max }}$ in Equation 1 and taking $\lambda_{\exp }=\lambda_{\log }$, the resulting equation is:

$$
\frac{d N(t)}{d t}=\lambda_{\log } N(t)\left(1-\frac{N(t)}{N_{\max }}\right)
$$

Equation 7 is the logistic equation written in the form of ODE. $N(t)$ denotes the total number of confirmed cases as a function of time, and the respective solution is easily found as follow:

$$
N(t)=\frac{N_{0} N_{\max } e^{\lambda_{\log } t}}{N_{\max }+N_{0}\left(e^{\lambda_{\log t}}-1\right)}
$$

Note that Equation 6 and Equation 8 can be used to estimate the $\lambda_{\log }$ and $N_{\max }$ parameters from the curve of the total case (Figure 1b), but the $t_{0}$ parameter cannot be estimated from Equation 8 . 
Now, using the difference logistic equation as follow:

$$
\frac{d N(t)}{d t}=N_{i+1}+N_{i}=K(t)
$$

Where $K(t)$ is the daily reported cases, the following expression is found:

$$
K(t)=\lambda_{\log } N(t)\left(1-\frac{N(t)}{N_{\max }}\right)
$$

Which shows a simple relation between $K(t)$ (daily cases) and $N(t)$ (total cases) (Figure 1c). Interestingly enough, Equation 10 can also be used to estimate the $\lambda_{\log }$ and $N_{\max }$ parameters. Additionally, the logistic growth rate can be found from Equation 10, as follow:

$$
\lambda_{\log }=\frac{K(t)}{N(t)\left(1-\frac{N(t)}{N_{\max }}\right)}
$$

In fact, Equation 11 can be used to study the growth rate variability as a function of total confirmed cases $(N(t))$ or time $(t)$. To simplify the notation, $\lambda_{\text {exp }}$ and $\lambda_{\log }$ have been taken as $\lambda$ through the text, Figures, and Tables.

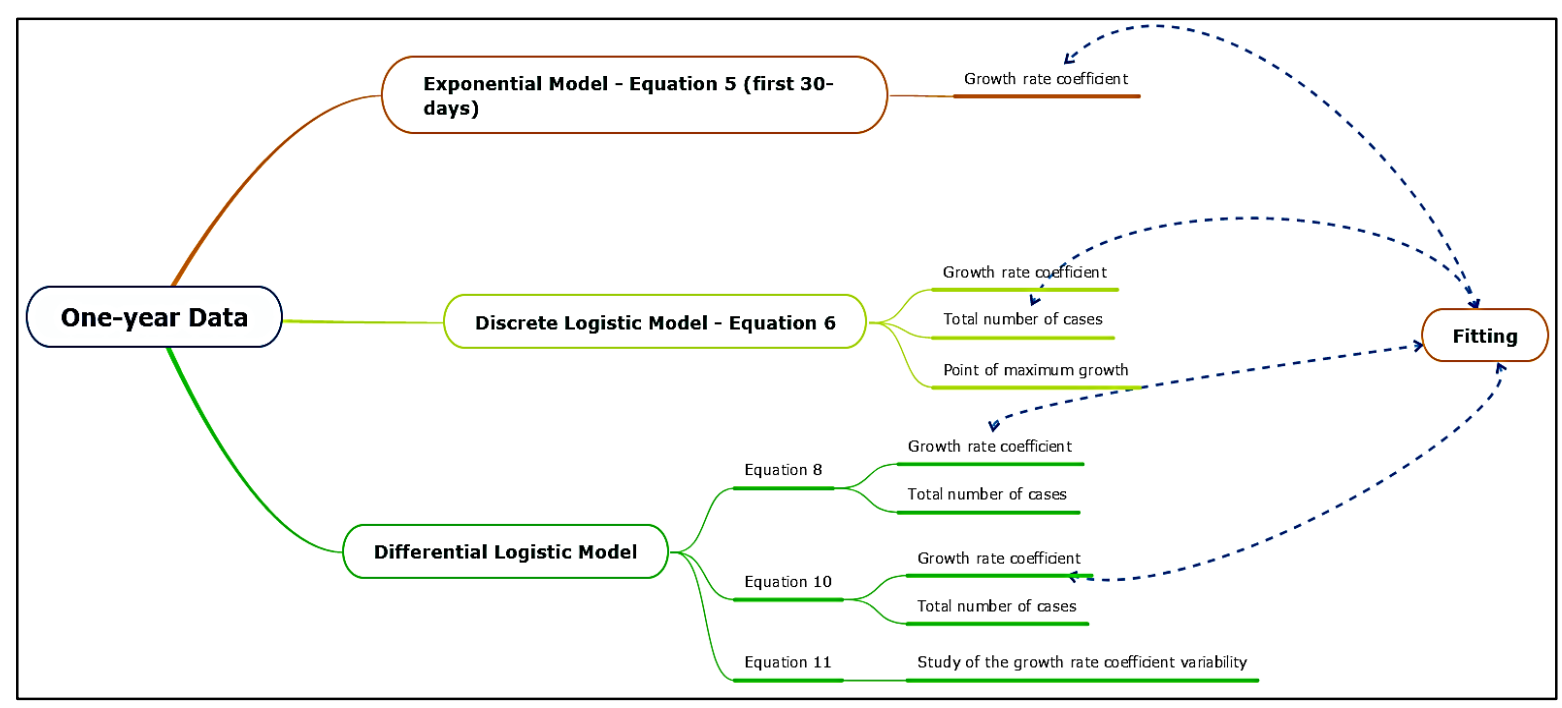

Figure 2. Schematic representation of the research methodology

Figure 2 shows the research methodology of the present work. In the exponential model, the data of the first 30 days were used, where an exponential increase in the number of infected people was observed. In the other models, the complete data set was analyzed with a seven-day moving average (359 data points). As observed, the exponential model gives access to the exponential growth rate at the beginning of the pandemic. On the other hand, the discrete logistic model helps correct the error of the exponential model for long periods, also allowing to estimate the logistic growth rate, the total number of infected people, and the inflection point of the curve. Most importantly, the differential logistic model can give a more complete picture of the COVID-19 pandemic because Equations 8 and 10 can be used to estimate the growth rate coefficient and the total number of infected people as a function of time or total cases, respectively. Finally, Equation 11 allows to study the growth rate coefficient variability, evidencing the random phenomenon of data as a consequence of the collecting/reporting data or social behavior.

\section{3- Results and Discussion}

Ecuador is a country in northwestern South America, bordered by Colombia on the north, Peru on the east and south, and the Pacific Ocean on the west. Ecuador also includes the Galápagos Islands. The largest cities are Quito and Guayaquil located in the provinces of Pichincha and Guayas, respectively. In terms of population density, Pichincha (333.08 people per $\mathrm{km}^{2}$ ) and Guayas (275.47 people per $\mathrm{km}^{2}$ ) have the highest number. The epicenter of the COVID-19 pandemic in Latin America was the city ofGuayaquil according to The Brazilian Reports (see: https://brazilian.report /liveblog/coronavirus/2020/07/26/ecuador-coronavirus-epicenter-moves-from-guayaquil-to-quito/). In particular, Guayaquil was the first city in Latin America to be completely devasted by the current pandemic due to several images of dead bodies lying on the streets of the Ecuadorian city shocked the world in March and April 2020. 


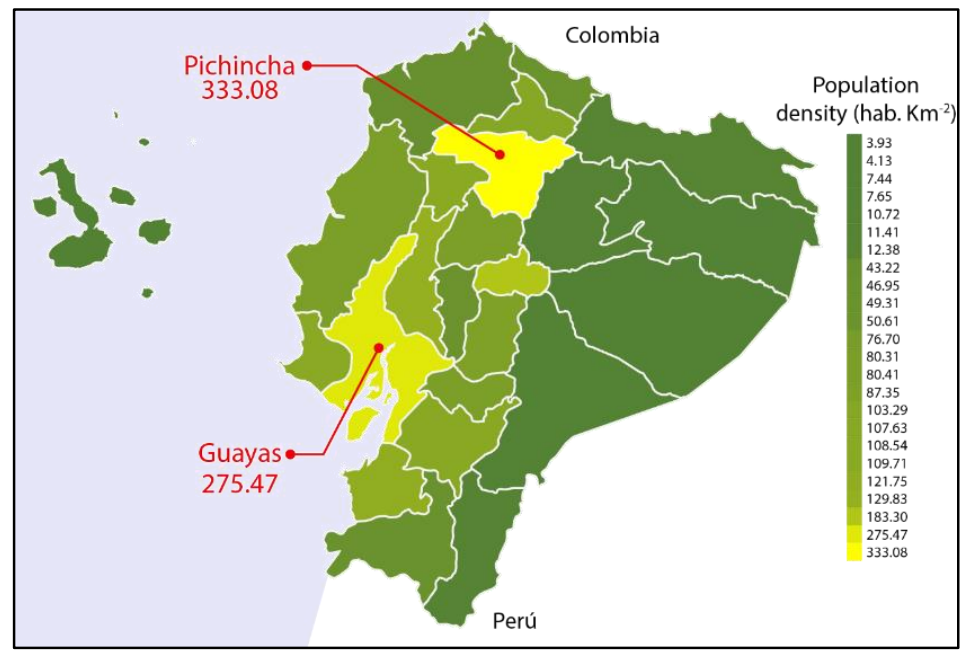

Figure 3. Ecuador population density map

The spread of COVID-19 in Ecuador is shown in Figure 4. Reported data are shown in the black color and 7-day moving average data in the red color. As mentioned earlier, the 7-day moving average dataset will be used throughout this work to avoid the peaks and valleys due to inadequate daily data collection and reporting. Although Ecuador had about two months to prepare for the pandemic, the health system and communication channels could not fulfill their purpose against the COVID-19 disease as observed in Figure 4a-4c and discussed below.
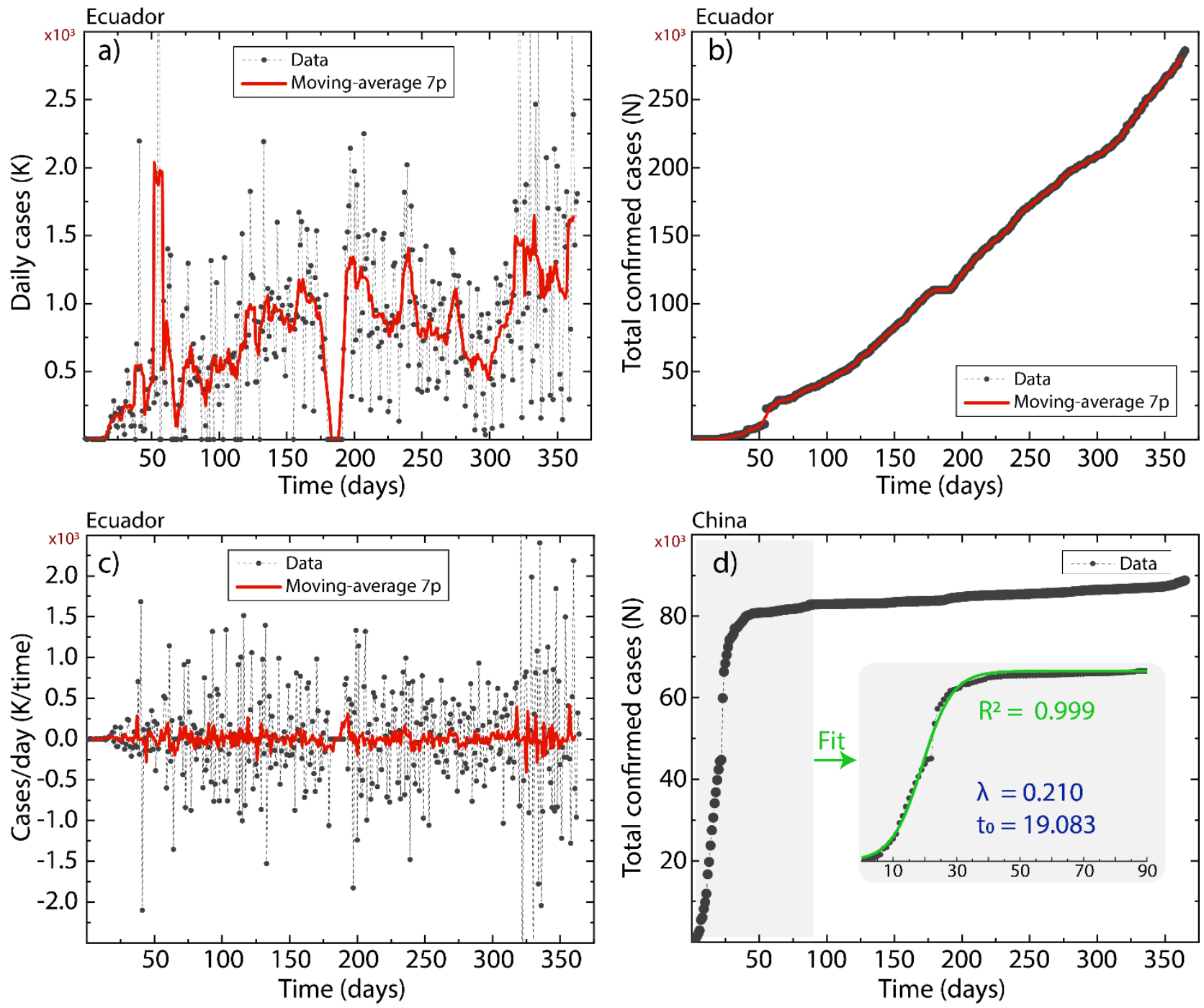

Figure 4. Ecuador: a) the number of infected people per day, b) the total number of confirmed cases reported on 02/28/2021, and c) the forward differential method applied to the daily reported cases. China: d) the total number of confirmed cases reported on 12/31/2020. Data are represented in black and the 7-point average data in red. The inset in Figure 1d shows the fit of China data using the discrete logistic model. 
Figure 4a shows the daily reported cases as a function of time within a 365-day window. As observed, the reported data are characterized by several intense peaks over the $1.5 \times 10^{3}$ daily cases. However, a deep valley is observed between days 180 and 190 (from August 27 to September 7, 2020), where zero new cases were reported. This strongly suggests the use of the 7-day moving average. Although the dispersion of the data points concerning the moving-average curve is noticeable, the trend of the data is maintained and the entire analysis can be continued with the 7-day movingaverage data. Taking this into account, the number of daily cases continues to increase after twelve months, confirming not an early saturation with current public health policies.

Build on the same data, the total number of confirmed cases increases with time (Figure $3 b$ ). In particular, an extra interruption in the reported data can be observed from May 4 to May 8, 2020 (days 65-69), which is corrected by the 7day moving average approach. Most importantly, the COVID-19 data from Ecuador contrast, for instance, with those reported by China where saturation of total infected people was achieved in approximately two months (at the beginning of March 2020) [30,31]. The latter is confirmed in Figure 4d. Nevertheless, the spread of COVID-19 in Ecuador follows almost the same scenario in all Latin American countries [32-34], which means an exponential increase in the number of cases, and the pandemic has not yet reached its peak. To identify the different waves in Ecuador, Figure 4c displays the forward differential method applied to the daily reported cases. However, a clear first or second wave or more cannot be evidenced because the data maintain a constant trend between $\pm 1.0 \times 10^{3}$, suggesting that the population continues to be infected without any control. The latter requires immediate intervention by state and local authorities in the adoption of restrictive regulations until the population has access to the vaccine.

Since there is currently no global solution to the pandemic, modeling is an excellent option to explore the effect of COVID-19 and predict its consequences. This can be done by estimating the (exponential or logistic) growth rate, the point of maximum growth, and the maximum number of infected people. In this context, Figure 5 shows the curve of the total case compared to different models: the exponential model (Figure 5a), the discrete logistic equation (Figure $5 \mathrm{~b}$ ), and the solution of the ODE logistic model (Figure 5c). The estimated value of the constants and corresponding statistical parameters (standard error and $\mathrm{R}^{2}$ ) are reported in Table 1.
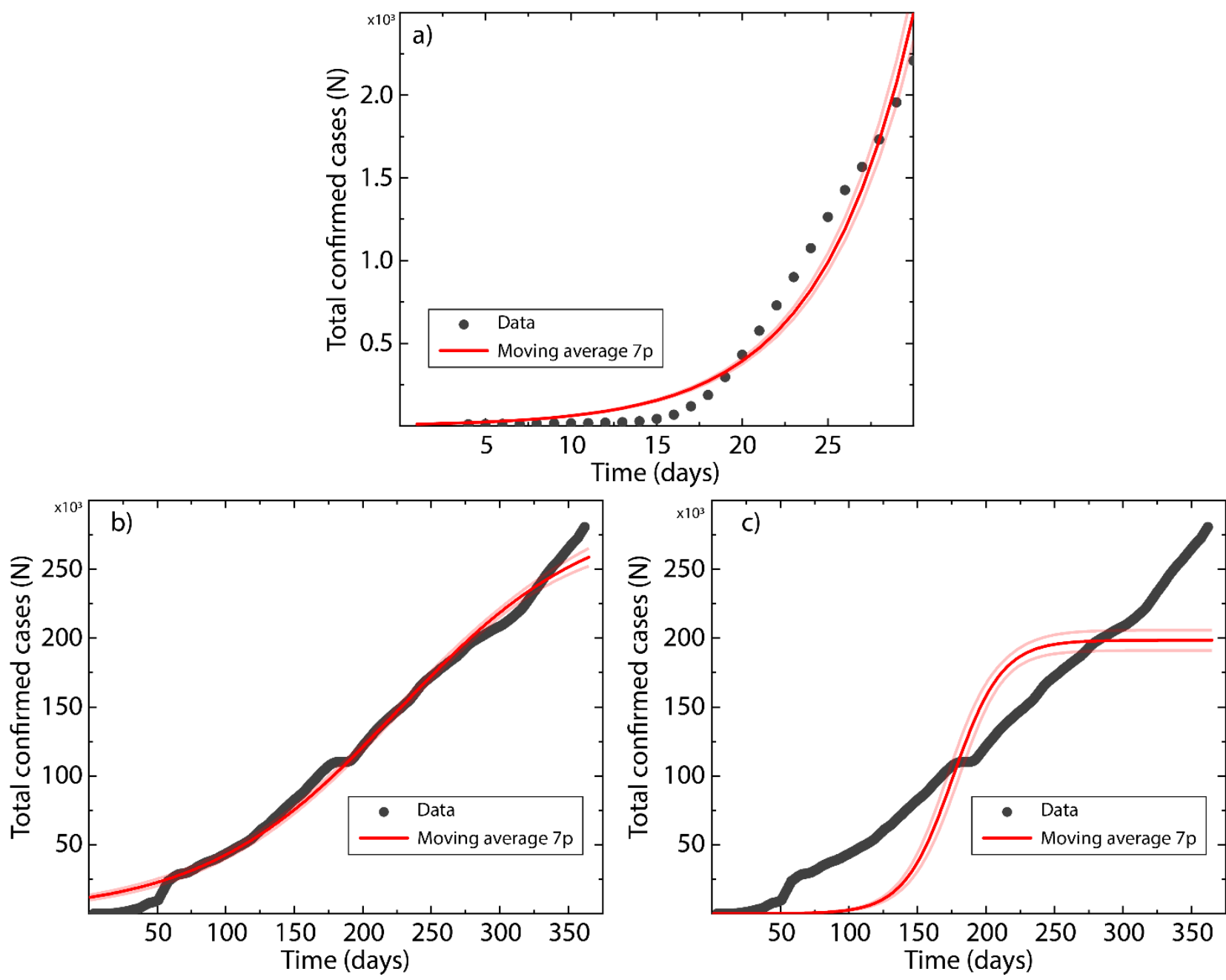

Figure 5. The total number of cases as a function of time fitted with different models: a) exponential model (Equation 5), b) discrete logistic equation (Equation 8), and c) solved ODE logistic equation (Equation 10). Data and fit are represented in black and red, respectively. Pink lines represent the confidence level (95\%). 
Table 1. Estimated parameters using the different models of the present work. The $\lambda$ represents the estimated (exponential or logistic) growth rate, $N_{\max }$ is the maximum number of infected people, and $t_{0}$ denotes the sigmoid midpoint.

\begin{tabular}{cccc}
\hline \multicolumn{4}{c}{ Exponential Model - Equation 5 } \\
\hline Parameter & Estimated & Standard Error & $\mathbf{R}^{\mathbf{2}}$ \\
$\lambda$ & 0.185 & 0.001 & 0.978 \\
\hline$\lambda$ & Discrete Logistic Model - Equation 6 & \\
$t_{0}$ & 0.014 & 0.001 & 0.997 \\
$N_{\max }$ & 225.563 & 2.231 & \\
\hline \multicolumn{5}{c}{ Solved ODE - Equation 8 } \\
\hline$N_{\max }$ & 294730 & 3597.460 & 0.915 \\
\hline
\end{tabular}

As observed, the growth rate coefficient estimated from the exponential model $(\lambda=0.185)$ is around 13.2 times and 3.3 times higher than those found by Equation $6(\lambda=0.014)$ and Equation $8(\lambda=0.056)$, respectively. The high growth rate of the exponential model is attributed to the fact that any control measure was applied at the beginning of the pandemic and health policies were not implemented on time. Such is the case that Ecuador suspected its first case of COVID-19 since February 14, 2020, but a partial quarantine was only imposed on March 17, 2020. Thus, on April 2, 2020 (two weeks after the official arrival of the pandemic in Latin America), the BBC showed that Ecuador ranked second in number of infected people and deaths per capital after Brazil (see: https://www.bbc.com/mundo/noticiasamerica-latina-52036460). On April 23, 2020, The New York Times reported that the total number of deaths was fifteen times more than those reported by the government, being the city of Guayaquil the most affected (see: https://www.nytimes.com/2020/04/23/world/americas/ecuador-deaths-coronavirus.html).

Figure 5a shows a clear exponential growth in the first 30 days with few points above or below the exponential fit (red line) and confidence interval (pink lines). Within the 30-days window, the total number of infected people was $N_{\max }=2240$ as of March 31, 2020. Most of the cases were reported in the province of Guayas $\left(N_{\max }=1615\right)$. On the other hand, the discrete logistic model shows an excellent agreement between the reported data and the fitting curve (Figure 5b). Comparing the midpoint values of Ecuador $\left(t_{0} \sim 226\right)$ and China $\left(t_{0} \sim 19\right.$, Figure $3 \mathrm{~d}$ inset), it is observed that the flattening of the infection curve in Ecuador began on October 12, 2020, more than seven months after the arrival of the pandemic. Furthermore, this value is 12 times higher than that reported by China where the numerical result suggests that the pandemic was controlled the first month. Most importantly, Figure $5 \mathrm{c}$ shows a discrepancy between the reported data and the fitting curve, suggesting that Equation 8 (the solution of the ODE differential logistic model) should be used with caution for long-term forecasting. In particular, the total number of infected people $\left(N_{\max }=\right.$ 203776) could be underestimated. The latter fails with almost 100000 cases of infected people compared to what is estimated by the discrete logistic model $\left(N_{\max }=294730\right)$. Beyond this, the approximation of the available data, reported in Table 1, is good enough $\left(R^{2}>0.8\right)$ [23], suggesting the approach proposed is sustainable for the Ecuador case, considering a complete data set of one year or more.

Now we focus on the estimated growth rate coefficient to qualitatively scrutinize Ecuador's policies against the COVID-19 disease. As public health strategies were implemented, for example, mitigation (social distancing, case testing, and symptomatic case isolation) and suppression (case isolation, quarantine, treatment of COVID-19 patients, and viral testing), the rate growth rate coefficient decreases, which means a reduction in the number of infected people over the months. This result is shown in Figure 6 where the possible contact of infected people is also illustrated. When analyzing the exponential model (Figure 6a) versus Equations 6 (Figure 6b) and Equation 8 (Figure 6c), the growth rate coefficient decreases by $\sim 92 \%$ and $\sim 70 \%$, respectively. Nevertheless, the growth rate coefficient estimated by Equation 8 is four times higher than that estimated by Equation 6, confirming a chaotic/random behavior as a function of time [23] (discussed below). This growth rate variation is attributed to government health rules taken at random, the negligence of the population, and a weak healthcare system. To all these, we must add the political meetings in the second half of 2020 (see: https://www.bbc.com/news/world-latin-america-56674200). In fact, Ecuador is characterized by a multi-stage behavior where the first, second, or more waves overlap (Figure 3c). To support this idea, it is necessary to mention that Ecuador ended the quarantine on May 4, 2020, proposing social distancing as the only rule. After this date, many holidays and festivities were celebrated without any control from the government and without reinforcing the health sector until now. 

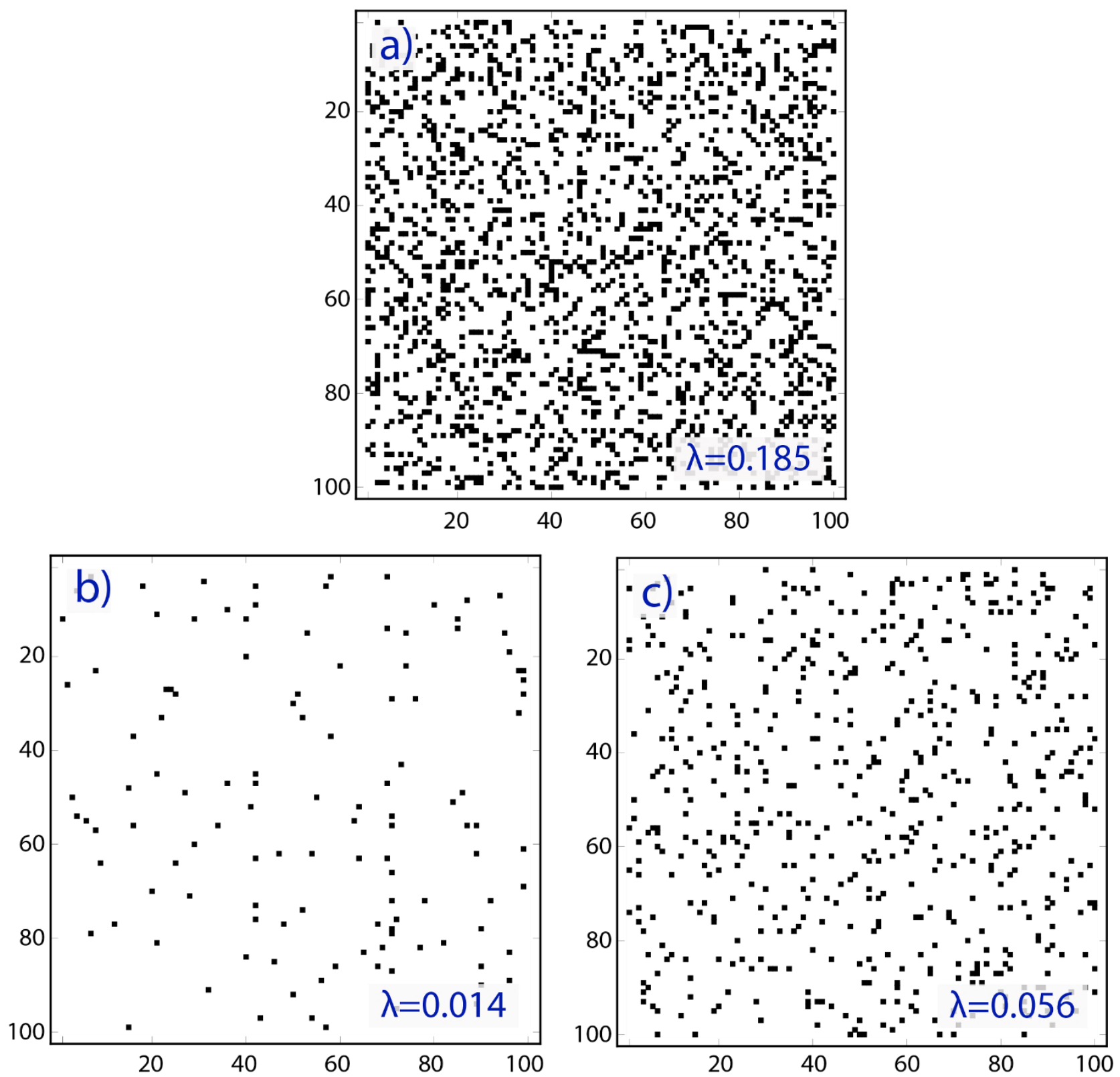

Figure 6. Matrix representation of the growth rate coefficient $(\lambda)$ from (a) exponential model (for simplicity is taken $\lambda$ ), (b) discrete logistic equation, and (c) solved ODE logistic model. Black markers represent the infected people affected by COVID19.

Let us stress again, the maximum number of infected people can be estimated either Equation $6\left(N_{\max }=294730\right)$ or Equation $8\left(N_{\max }=203776\right)$ but the resulting values were exceeded on March 9, $2021\left(N_{\max }=295114\right)$ and December $17,2020\left(N_{\max }=204249\right)$, respectively. These results evidence that the discrete logistic model and the solution of the ODE logistic model cannot be used to properly predict the total number of cases when a sigmoidal trend is not observed, such as that reported in Figure $4 \mathrm{~d}$ inset. With this in mind, a more accurate value of the maximum number of infected people can be predicted by means of Equation 10. This equation shows a simple relationship between daily reported cases $(K)$ and total cases $(N)$ but allows calculating the growth rate coefficient and the maximum number of cases with current state policies. For comparative purposes, Equation 10 has also been applied to Brazil, Peru, and Colombia. These countries show the same behavior as Ecuador (Figure 4b). The estimated values and corresponding statistical parameters are reported in Table 2. In Figure 7, the parabolic approximation curves (red) show a reasonable agreement with the data reported for the different countries $\left(R^{2}>0.80\right)$ [23], giving a $N_{\max }=409156$ for Ecuador, $N_{\max }=1.606 \times 10^{7}$ for Brazil, $N_{\max }=1.579 \times 10^{6}$ for Peru, and $N_{\max }=2.827 \times 10^{6}$ for Colombia. The estimated $N_{\max }$ value in Ecuador agrees with the data reported at the end of May $2021\left(N_{\max }=426037\right)$ (see the following link for the real-time updated map on the Ecuador situation of cases of coronavirus: https://coronavirus.jhu.edu/region/ecuador). This suggests that the proposed logistic model may be useful and further calibrated if more data from daily cases are added. 

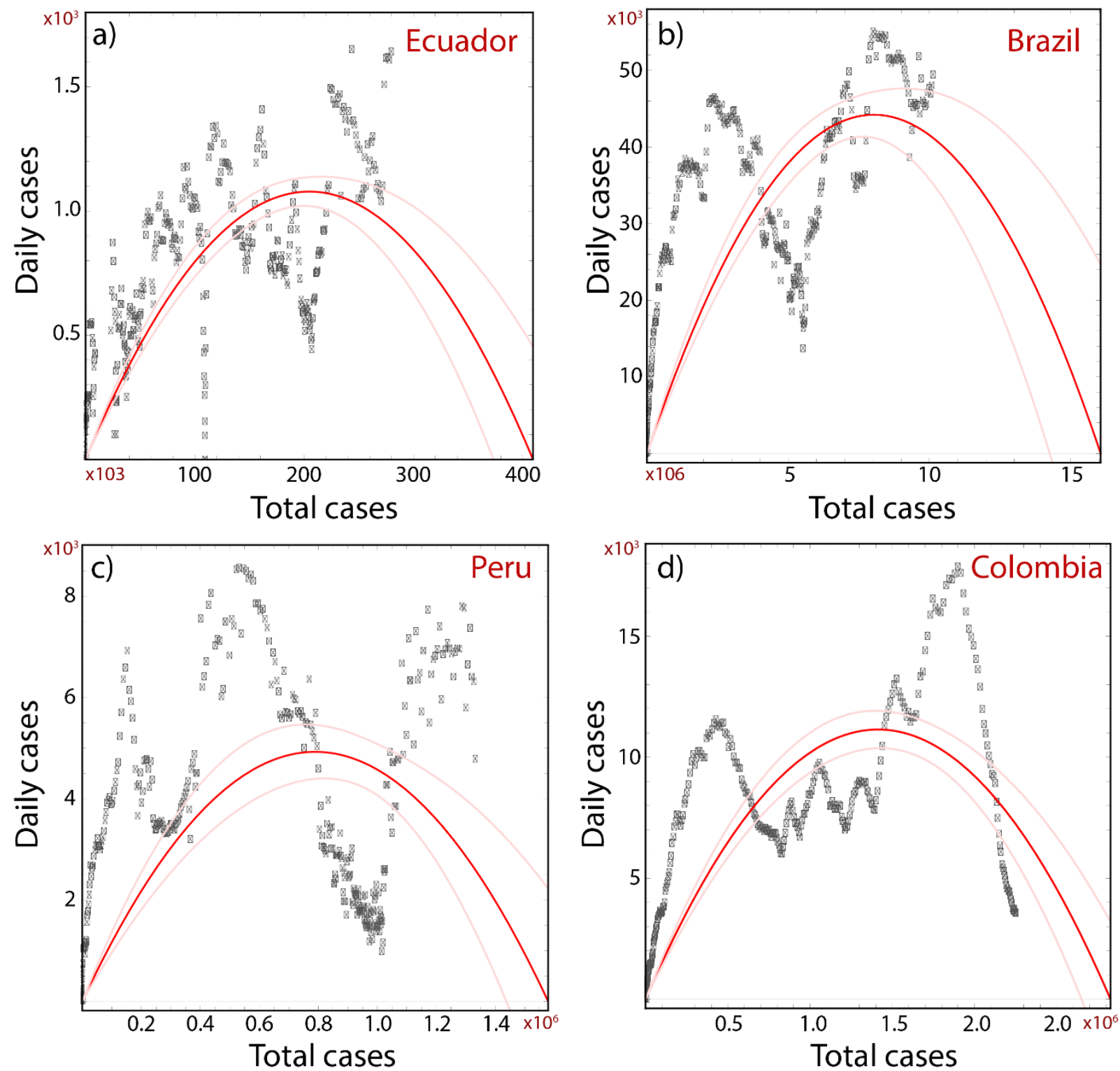

Figure 7. The daily cases as a function of the total confirmed cases. The black markers show the data and the red curve is the regression according to Equation 10 . Pink lines represent the confidence level $(95 \%)$. For comparison, it is analyzed different countries: a) Ecuador, b) Brazil, c) Peru, and d) Colombia.

Table 2. Estimated parameters using Equation 10 applied to different countries: Ecuador, Brazil, Peru, and Colombia.

\begin{tabular}{|c|c|c|c|}
\hline \multicolumn{4}{|c|}{ Equation 10} \\
\hline Parameter & Estimated & Standard Error & $\mathbf{R}^{2}$ \\
\hline \multicolumn{4}{|c|}{ Ecuador } \\
\hline$\lambda$ & 0.011 & 0.001 & \multirow{2}{*}{0.902} \\
\hline$N_{\max }$ & 409156 & 2922.990 & \\
\hline \multicolumn{4}{|c|}{ Brazil } \\
\hline$\lambda$ & 0.011 & 0.001 & \multirow{2}{*}{0.856} \\
\hline$N_{\max }$ & $1.606 \times 10^{7}$ & $1.139 \times 10^{6}$ & \\
\hline \multicolumn{4}{|c|}{ Peru } \\
\hline$\lambda$ & 0.013 & 0.001 & \multirow{2}{*}{0.905} \\
\hline$N_{\max }$ & $1.579 \times 10^{6}$ & 90730.921 & \\
\hline \multicolumn{4}{|c|}{ Colombia } \\
\hline$\lambda$ & 0.016 & 0.001 & \multirow{2}{*}{0.897} \\
\hline$N_{\max }$ & $2.827 \times 10^{6}$ & 61098.893 & \\
\hline
\end{tabular}


Interestingly enough, the growth rate estimated by Equation 10 for Ecuador $\left(\lambda=0.011\right.$ day $\left.^{-1}\right)$ shows a decrease of $\sim 94 \%$ compared to that found in the exponential model $\left(\lambda=0.185 \mathrm{day}^{-1}\right)$. This result agrees with that reported by the discrete logistic model $\left(\lambda=0.014\right.$ day $\left.^{-1}\right)$. Furthermore, this result is similar to that found in Brazil $\left(\lambda=0.011\right.$ day $\left.^{-1}\right)$ but slightly lower than that reported in Peru $\left(\lambda=0.013\right.$ day $\left.^{-1}\right)$ and Colombia $\left(\lambda=0.016\right.$ day $\left.^{-1}\right)$, confirming the same scenario of the Ecuadorian case as well as that the government policies and the social behavior adopted could be very similar in these countries. As observed in the different countries, the scatter of data points concerning the parabolic curve and the confidence interval is not small, indicating that it is necessary to consider the variability of the growth rate coefficient as a time function, mainly, if new daily cases are reported. The coefficient variability can be determined from Equation 11, expressed as a function of total cases (Figure 8) or time (Figure 9). We focus on the Ecuadorian case, which is the main goal of the present work. However, similar results were found in the other countries considered here.
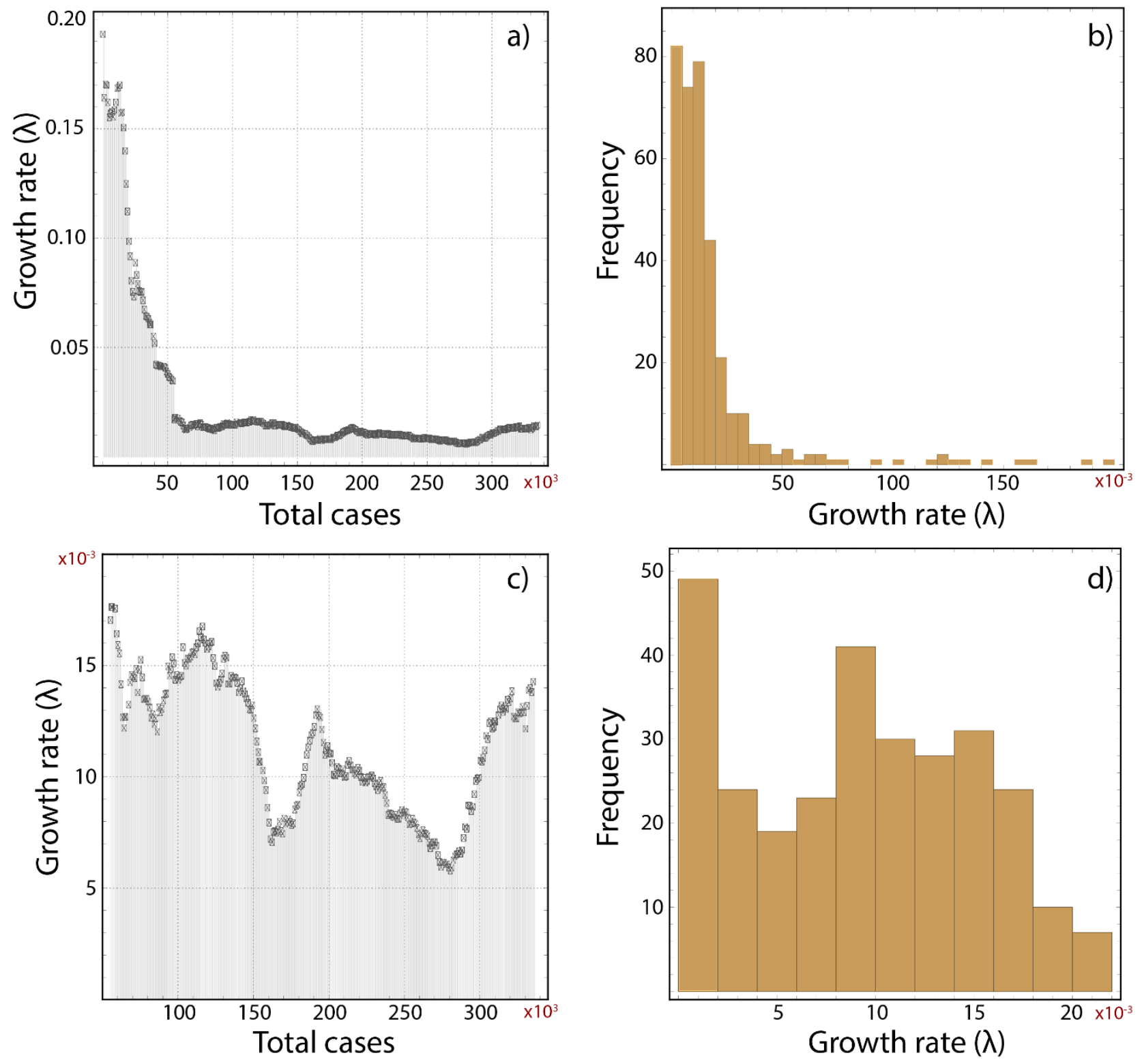

Figure 8. a), c) the growth rate coefficient as a function of the total confirmed cases computed using Equation 11 and considering the data in a one-year window for Ecuador. b), d) the associated histograms.

Figure 8 shows the variability of the coefficient $\lambda$. Figure 8 a shows that the growth rate coefficient obtained the highest values in the first $10 \times 10^{3}$ total confirmed cases $(0.2<\lambda<0.08)$. After $20 \times 10^{3}$ and $30 \times 10^{3}$ total confirmed cases, the value of the coefficient $\lambda$ decreases to $\sim 0.05$ and $\sim 0.01$, respectively. The variability of the coefficient $\lambda$ is confirmed in Figure $8 \mathrm{c}$, from $40 \times 10^{3}$ to $300 \times 10^{3}$ total cases with $0.02<\lambda<0.005$. A close picture shows three broad peaks as the number of total cases increase. The first peak between $80 \times 10^{3}$ and $160 \times 10^{3}$, the second peak between $170 \times 10^{3}$ and $280 \times 10^{3}$, and the third peak from $290 \times 10^{3}$. These peaks are related to a multi-stage COVID-19 outbreak in Ecuador, and not necessarily to several waves. The maximum of the first and second peaks is found in the week of September 28 to October 4, 2020, and December 7 to 13, 2020. In particular, the second peak coincides with the beginning of the Christmas and New Year celebrations. 
Figure $8 \mathrm{~b}$ shows a skewed right, i.e., the distribution of the growth rate coefficient is asymmetric with a tail going off to the right. On the right side, there are few growth rates whose values are higher than the rest. Most of the growth rate coefficients are found below $0.02 \mathrm{day}^{-1}$. The latter is corroborated in Figure 8d, where an almost symmetrical characteristic can be observed, and most of the growth rates coefficients are found between 0.007 and 0.017 days $^{-1}$. The highest frequency value is found at a growth rate coefficient of $0.001 \mathrm{day}^{-1}$.
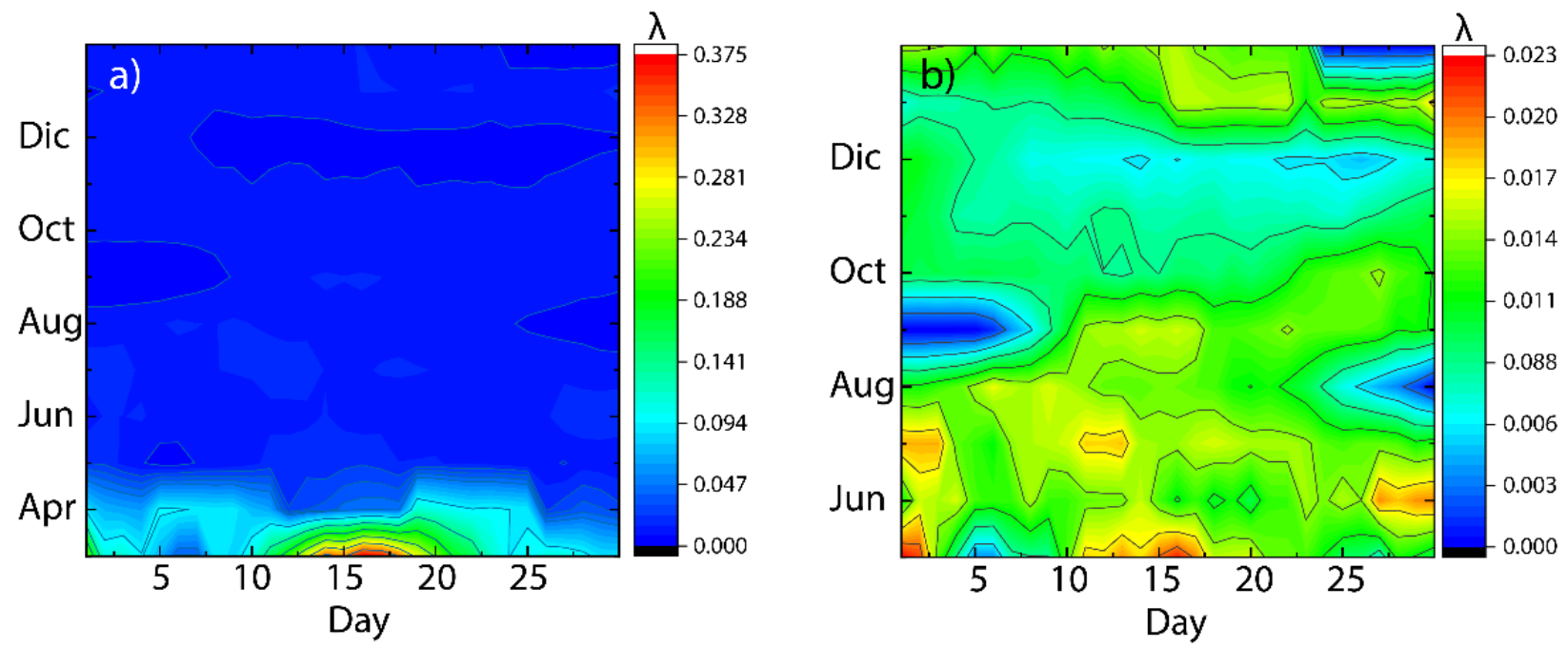

Figure 9. The growth rate coefficient of Equation 11 is represented as a surface-color plot vs 30-days and 12-months. (a) the growth rate in a one-year window and (b) the growth rate from May 1, 2020, to February 28, 2021.

Finally, Figure 9 displays the growth rate coefficient $(\lambda)$ as a function of time. As observed, March and April 2020 were the most critical months in Ecuador, mainly, the maximum values of the coefficient $\lambda$ are observed in the third week (Figure 9a). In the remaining months (from May 2020 to February 2021), a variability of the coefficient $\lambda$ is also appreciated (Figure 9b) which can be attributed to the variable number of examined people per day or social behavior [35]. Nevertheless, its values are not as high as in the first months because the population adopted for personal care, such as face masks, sanitizers, social distancing, and limited intercity mobility.

\section{4- Conclusion}

Here, we have presented a simple mathematical approach based on the conventional exponential model, the discrete logistic equation, and the differential logistic model to scrutinize the COVID-19 outbreak in Ecuador. The proposed mathematical approach is partially extended to Brazil, Peru and Colombia. The estimated parameters were the growth rate $(\lambda)$, the total number of cases $(N)$, and the midpoint of the maximum growth $\left(t_{0}\right)$. Additionally, the variability of the growth rate coefficient was study as a function of the total cases or time. The one-year data from March 1, 2020, to February 28, 2021 were analyzed considering a seven-point moving average. The results evidenced that the growth rate decreased from $\lambda=0.185$ day $^{-1}$ (exponential model) to $\lambda=0.014$ day $^{-1}$ (discrete model) and $\lambda=0.056$ day $^{-1}$ (differential model) over the months. While the lockdown declared on March 17, 2020, in Ecuador had a "relative" positive effect on reducing the growth rate values, the high number of infected people in the early days of the pandemic collapsed Ecuador's weak health service. The latter is corroborated by analyzing the growth rate variability as function of time where it is observed that the most critical months of the pandemic were March and April 2020. The total number of cases were estimated by discrete model $(N \sim 295 \mathrm{~K})$ and differential model $(N \sim 204 \mathrm{~K})$, showing that neither of them can be used for long-term forecasting, given that the first one fits well to one-year data and the second the total number of cases is underestimated. However, this problem can be solved by analyzing daily cases as a function of total cases. Thus, the predicted numerical value $(N \sim 409 \mathrm{~K})$ of the total number of infected people was found to be in good agreement with the real-time data reported at the end of May 2021. This simple mathematical approach can be calibrated by adding the daily data of infected people, and it can be used in other Latin American countries and localities to take immediate action against the COVID-19 disease.

\section{5- Declarations}

\section{5-1-Author Contributions}

Conceptualization, supervision, resources, T.T., J.S., R.T., and C.V.G.; methodology, validation, formal analysis, C.V.G, and T.T.; data curation, visualization, M.G.; writing — original draft preparation, C.V.G.; writing-review and editing, C.V.G. All authors have read and agreed to the published version of the manuscript. 


\section{5-2-Data Availability Statement}

The data that supports the findings of this study are available within the article. Any additional data relevant to this study are available from the author upon reasonable request.

\section{5-3- Funding}

This work has been funding by Universidad Técnica Particular de Loja (UTPL-Ecuador).

\section{5-4-Acknowledgements}

T.T. thanks Universidad Técnica Particular de Loja for supporting the present work. This work is in memory of Pedro Gómez, who died from COVID-19 during the development of this research.

\section{5-5- Conflicts of Interest}

The authors declare that there is no conflict of interests regarding the publication of this manuscript. In addition, the ethical issues, including plagiarism, informed consent, misconduct, data fabrication and/or falsification, double publication and/or submission, and redundancies have been completely observed by the authors.

\section{6- References}

[1] Manabe, Toshie, Hiroyasu Akatsu, Kazuhiko Kotani, and Koichiro Kudo. "Trends in Clinical Features of Novel Coronavirus Disease (COVID-19): A Systematic Review and Meta-Analysis of Studies Published from December 2019 to February 2020." Respiratory Investigation 58, no. 5 (September 2020): 409-418. doi:10.1016/j.resinv.2020.05.005.

[2] Ikeuchi, Kazuhiko, Makoto Saito, Shinya Yamamoto, Hiroyuki Nagai, and Eisuke Adachi. "Relative Bradycardia in Patients with Mild-to-Moderate Coronavirus Disease, Japan.” Emerging Infectious Diseases 26, no. 10 (October 2020): 2504-2506. doi:10.3201/eid2610.202648.

[3] Hu, Ben, Hua Guo, Peng Zhou, and Zheng-Li Shi. "Characteristics of SARS-CoV-2 and COVID-19." Nature Reviews Microbiology 19, no. 3 (October 6, 2020): 141-154. doi:10.1038/s41579-020-00459-7.

[4] Gragnani, L., M. Monti, S. A. Santini, S. Marri, F. Madia, S. Lorini, L. Petraccia et al. "SARS-CoV-2 was already circulating in Italy, in early December 2019." European Review for Medical and Pharmacological Sciences 25, no. 8 (2021): 3342-3349. doi: 10.26355/EURREV_202104_25746.

[5] W. H. Organization and others, "COVID 19 Public Health Emergency of International Concern (PHEIC). Global research and innovation forum: towards a research roadmap," 2020.

[6] Del Guayo Castiella, Iñigo, and Miguel A Marmolejo Cervantes. "The Recovery of the Energy Sector after the COVID-19 Pandemic: a Comparison between Latin America and the European Union.” Journal of Energy \& Natural Resources Law (June 23, 2021): 1-17. doi:10.1080/02646811.2021.1930710.

[7] Franz, Tobias. "Spatial Fixes and Switching Crises in the Times of COVID-19: Implications for Commodity-Producing Economies in Latin America." Canadian Journal of Development Studies / Revue Canadienne D'études Du Développement 42, no. 1-2 (October 22, 2020): 109-121. doi:10.1080/02255189.2020.1832881.

[8] Alava, Juan José, and Angel Guevara. "A Critical Narrative of Ecuador's Preparedness and Response to the COVID-19 Pandemic." Public Health in Practice 2 (November 2021): 100127. doi:10.1016/j.puhip.2021.100127.

[9] Torres, Irene, Rachel Sippy, and Fernando Sacoto. "Assessing Critical Gaps in COVID-19 Testing Capacity: The Case of Delayed Results in Ecuador.” BMC Public Health 21, no. 1 (April 1, 2021). doi:10.1186/s12889-021-10715-x.

[10] Cifuentes-Faura, Javier. "COVID-19 Mortality Rate and Its Incidence in Latin America: Dependence on Demographic and Economic Variables.” International Journal of Environmental Research and Public Health 18, no. 13 (June 27, 2021 ): 6900. doi:10.3390/ijerph18136900.

[11] Ruiz-Patiño, Alejandro, Oscar Arrieta, Luis E. Pino, Christian Rolfo, Luisa Ricaurte, Gonzalo Recondo, Zyanya-Lucia ZatarainBarron, et al. "Mortality and Advanced Support Requirement for Patients With Cancer With COVID-19: A Mathematical Dynamic Model for Latin America.” JCO Global Oncology no. 6 (November 2020): 752-760. doi:10.1200/go.20.00156.

[12] Espinosa, Pablo, Paulina Quirola-Amores, and Enrique Teran. “Application of a Susceptible, Infectious, And/or Recovered (SIR) Model to the COVID-19 Pandemic in Ecuador.” Frontiers in Applied Mathematics and Statistics 6 (November 30, 2020). doi:10.3389/fams.2020.571544.

[13] Katal, Sanaz, Lee Myers, and Ali Gholamrezanezhad. "SARS-CoV-2 Reinfection: 'New Baseline' Imaging Concept in the Era of COVID-19.” Clinical Imaging 78 (October 2021): 142-145. doi:10.1016/j.clinimag.2021.03.021.

[14] Dos Santos, Wagner Gouvêa. "Impact of Virus Genetic Variability and Host Immunity for the Success of COVID-19 Vaccines." Biomedicine \& Pharmacotherapy 136 (April 2021): 111272. doi:10.1016/j.biopha.2021.111272. 
[15] Mackolil, Joby, and Basavarajappa Mahanthesh. "Logistic Growth and SIR Modelling of Coronavirus Disease (COVID-19) Outbreak in India: Models Based on Real-Time Data." Mathematical Modelling of Engineering Problems 7, no. 3 (September 30, 2020): 345-350. doi:10.18280/mmep.070303.

[16] Furlan, Claudia, and Cinzia Mortarino. "The Effect of Swabs on Modeling the First Wave of the COVID-19 Pandemic in Italy." Emerging Science Journal 5 (May 29, 2021): 37-61. doi:10.28991/esj-2021-sper-04.

[17] He, Shaobo, Yuexi Peng, and Kehui Sun. "SEIR Modeling of the COVID-19 and Its Dynamics." Nonlinear Dynamics 101, no. 3 (June 18, 2020): 1667-1680. doi:10.1007/s11071-020-05743-y.

[18] Matthew, Ekum, and Ogunsanya Adeyinka. "Application of Hierarchical Polynomial Regression Models to Predict Transmission of COVID-19 at Global Level." International Journal of Clinical Biostatistics and Biometrics 6, no. 1 (June 27, 2020). doi:10.23937/2469-5831/1510027.

[19] Lalmuanawma, Samuel, Jamal Hussain, and Lalrinfela Chhakchhuak. "Applications of Machine Learning and Artificial Intelligence for Covid-19 (SARS-CoV-2) Pandemic: A Review.” Chaos, Solitons \& Fractals 139 (October 2020): 110059. doi:10.1016/j.chaos.2020.110059.

[20] Larsen, Joseph R., Margaret R. Martin, John D. Martin, Peter Kuhn, and James B. Hicks. "Modeling the Onset of Symptoms of COVID-19." Frontiers in Public Health 8 (August 13, 2020). doi:10.3389/fpubh.2020.00473.

[21] Singhal, Amit, Pushpendra Singh, Brejesh Lall, and Shiv Dutt Joshi. "Modeling and Prediction of COVID-19 Pandemic Using Gaussian Mixture Model.” Chaos, Solitons \& Fractals 138 (September 2020): 110023. doi:10.1016/j.chaos.2020.110023.

[22] Moein, Shiva, Niloofar Nickaeen, Amir Roointan, Niloofar Borhani, Zarifeh Heidary, Shaghayegh Haghjooy Javanmard, Jafar Ghaisari, and Yousof Gheisari. "Inefficiency of SIR Models in Forecasting COVID-19 Epidemic: a Case Study of Isfahan." Scientific Reports 11, no. 1 (February 25, 2021). doi:10.1038/s41598-021-84055-6.

[23] Pelinovsky, Efim, Andrey Kurkin, Oxana Kurkina, Maria Kokoulina, and Anastasia Epifanova. "Logistic Equation and COVID19.” Chaos, Solitons \& Fractals 140 (November 2020): 110241. doi:10.1016/j.chaos.2020.110241.

[24] Wang, Peipei, Xinqi Zheng, Jiayang Li, and Bangren Zhu. "Prediction of Epidemic Trends in COVID-19 with Logistic Model and Machine Learning Technics." Chaos, Solitons \& Fractals 139 (October 2020): 110058. doi:10.1016/j.chaos.2020.110058.

[25] Abusam, Abdallah, Razan Abusam, and Bader Al-Anzi. "Adequacy of Logistic Models for Describing the Dynamics of COVID19 Pandemic.” Infectious Disease Modelling 5 (2020): 536-542. doi:10.1016/j.idm.2020.08.006.

[26] Gao, Xiang, and Qunfeng Dong. “A Logistic Model for Age-Specific COVID-19 Case-Fatality Rates.” JAMIA Open 3, no. 2 (April 13, 2020): 151-153. doi:10.1093/jamiaopen/ooaa025.

[27] Aviv-Sharon, Elinor, and Asaph Aharoni. "Generalized Logistic Growth Modeling of the COVID-19 Pandemic in Asia." Infectious Disease Modelling 5 (2020): 502-509. doi:10.1016/j.idm.2020.07.003.

[28] Livadiotis, George. "Statistical Analysis of the Impact of Environmental Temperature on the Exponential Growth Rate of Cases Infected by COVID-19." Edited by Oscar Millet. PLOS ONE 15, no. 5 (May 29, 2020): e0233875. doi:10.1371/journal.pone.0233875.

[29] Consolini, Giuseppe, and Massimo Materassi. “A Stretched Logistic Equation for Pandemic Spreading." Chaos, Solitons \& Fractals 140 (November 2020): 110113. doi:10.1016/j.chaos.2020.110113.

[30] Lau, Hien, Veria Khosrawipour, Piotr Kocbach, Agata Mikolajczyk, Justyna Schubert, Jacek Bania, and Tanja Khosrawipour. "The Positive Impact of Lockdown in Wuhan on Containing the COVID-19 Outbreak in China." Journal of Travel Medicine 27, no. 3 (March 17, 2020). doi:10.1093/jtm/taaa037.

[31] Vasconcelos, Giovani L., Antônio M. S. Macêdo, Gerson C. Duarte-Filho, Arthur A. Brum, Raydonal Ospina, and Francisco A. G. Almeida. "Power Law Behaviour in the Saturation Regime of Fatality Curves of the COVID-19 Pandemic." Scientific Reports 11, no. 1 (February 25, 2021). doi:10.1038/s41598-021-84165-1.

[32] Del Brutto, Oscar H, Aldo F Costa, Robertino M Mera, Bettsy Y Recalde, Javier A Bustos, and Héctor H García. "SARS-CoV2 in Rural Latin America. A Population-Based Study in Coastal Ecuador.” Clinical Infectious Diseases 73, no. 2 (July 27, 2020): 314-317. doi:10.1093/cid/ciaa1055.

[33] Miller, Matthew J., Jose R. Loaiza, Anshule Takyar, and Robert H. Gilman. "COVID-19 in Latin America: Novel Transmission Dynamics for a Global Pandemic?” Edited by Laith Yakob. PLOS Neglected Tropical Diseases 14, no. 5 (May 7, 2020): e0008265. doi:10.1371/journal.pntd.0008265.

[34] Zhu, Dongshan, Shiva Raj Mishra, Xikun Han, and Karla Santo. "Social Distancing in Latin America during the COVID-19 Pandemic: An Analysis Using the Stringency Index and Google Community Mobility Reports.” Journal of Travel Medicine 27, no. 8 (July 30, 2020). doi:10.1093/jtm/taaa125.

[35] Larios-Gómez, Emigdio, Laura Fischer, Mónica Peñalosa, and Mayra Ortega-Vivanco. "Purchase Behavior in COVID-19: A Cross Study in Mexico, Colombia, and Ecuador.” Heliyon 7, no. 3 (March 2021): e06468. doi:10.1016/j.heliyon.2021.e06468. 